\title{
INVARIANCE OF THE HOMOLOGY OF A LATTICE ${ }^{1}$
}

\author{
JOHN MATHER
}

G.-C. Rota, D. Kan, F. Peterson, and G. Whitehead have constructed a homology theory for finite lattices. Their definition is given in terms of a choice of "cross-cut" for the given lattice; however it was conjectured by G.-C. Rota [4, p. 356] that the Betti numbers so obtained are independent of the choice of cross-cut. It is an immediate consequence of the theorem proved in this paper that this is so. Jon Folkman [2] has given an independent proof of the invariance of the homology of a lattice. He shows that the homology of any cross-cut is the same as the homology of the complex whose vertices are the elements of the lattice other than 0 or 1 and whose simplices are the totally ordered subsets of the vertices. His proof is valid for infinite as well as finite lattices.

As in [4], a cross-cut of a finite lattice is a subset $C$ of $L$ such that:

(a) $C$ contains neither 0 nor 1 ,

(b) no two elements of $C$ are comparable,

(c) every chain stretched from 0 to 1 meets $C$.

A spanning subset of $L$ is a subset whose join is 1 and whose meet is 0 . Given a cross-cut $C$ of $L$, we define a simplicial complex $K(C, L)$. The vertices of $K(C, L)$ are the elements of $C$. The simplices of $K(C, L)$ are the subsets of $C$ which do not span $L$.

Theorem. If $C$ and $C^{\prime}$ are two cross-cuts of $L$, then $K(C, L)$ and $K\left(C^{\prime}, L\right)$ have the same homotopy type.

Let $U_{L}$ (resp. $V_{L}$ ) denote the cross-cut of $L$ whose elements are the maximal (resp. minimal) elements in $L-\{0,1\}$. If $C$ is any crosscut of $L$, we denote the first barycentric subdivision of $K(C, L)$ by $K^{\prime}(C, L)$, and define a map $f_{C, L}$ from the set of vertices of $K^{\prime}(C, L)$ to the set of vertices of vertices of $K^{\prime}\left(U_{L}, L\right)$ as follows. If $S$ is a vertex of $K^{\prime}(C, L)$ (in other words, a simplex of $K(C, L)$, i.e., a subset of $C$ which does not span $L$ ) then

$$
\begin{aligned}
f_{C, L}(S) & =\left\{x \in U_{L}: x \geqq \bigvee S\right\} & & \text { if } \vee S \neq 1, \\
& =\left\{x \in U_{L}: x \geqq \wedge S\right\} & & \text { if } \vee S=1 .
\end{aligned}
$$

To show that $f_{C, L}(S)$ is really a vertex of $K^{\prime}\left(U_{L}, L\right)$, we must show that it does not span $L$. If $\bigvee S \neq 1$ then $\Lambda f_{C, L}(S)>\vee S>0$. If $\bigvee S=1$

Received by the editors December 17, 1964.

1 This work was supported by the National Bureau of Standards. 
then, since $S$ does not span, $\Lambda S>0$. Hence, $\Lambda f_{c, L}(S) \geqq \Lambda S>0$.

We will prove our theorem by showing that $f_{C, L}$ is a simplicial map and that its geometric realization (which will also be denoted by $\left.f_{C, L}\right)$ is a homotopy equivalence.

LEMMA 1. $f_{C, L}$ is a simplicial map.

Proof. It suffices to show that if $S$ and $T$ are simplices of $K(C, L)$ with $S \subseteq T$, then either $f_{C, L}(S) \subseteq f_{C, L}(T)$ or $f_{C, L}(T) \subseteq f_{C, L}(S)$. There are two cases. If $\mathrm{V} T<1$, then $\mathrm{V} S<1$ and it follows from $\mathrm{V} S \leqq \mathrm{~V} T$ that $f_{C, L}(T) \subseteq f_{C, L}(S)$. If $\bigvee T=1$ then $x \in f_{C, L}(S)$ implies $x \geqq \Lambda S \geqq \Lambda T$ and hence $x \in f_{C, L}(T)$. Hence $f_{C, L}(S) \subseteq f_{C, L}(T)$ in this case.

The proof that $f_{C, L}$ is a homotopy equivalence will be by induction on

$$
p(C, L)=\operatorname{card}\{x \in L: \exists y \in C, x<y\} .
$$

Basis for the induction: $p(C, L)=1$. (This is a special case of Theorem 1 of [1].) In this case $C=V_{L}$, and the $\operatorname{map} f=f_{C, L}$ is given by

$$
f(S)=\left\{x \in U_{L}: x \geqq \vee S\right\}
$$

for any vertex $S$ of $K^{\prime}\left(V_{L}, L\right)$. Dually there is a simplicial map

$$
g: K^{\prime}\left(U_{L}, L\right) \rightarrow K^{\prime}\left(V_{L}, L\right)
$$

given by

$$
g(S)=\left\{x \in V_{L}: x \leqq \wedge S\right\}
$$

for any vertex $S$ of $K^{\prime}\left(U_{L}, L\right)$.

Observe that there is a partial order $(\leqq)$ on the vertices of the barycentric subdivision $K^{\prime}$ of any simplicial complex $K$, induced by the inclusion relation between simplices of $K$ via the correspondence between simplices of $K$ and vertices of $K^{\prime}$. In this notation it is easily seen that if $S$ and $T$ are vertices of $K^{\prime}\left(U_{L}, L\right)$ and $S \leqq T$ then

$$
S \leqq f g(S) \leqq f g(T) .
$$

We define a homotopy

$$
h:\left|K^{\prime}\left(U_{L}, L\right)\right| \times I \rightarrow\left|K\left(U_{L}, L\right)\right|
$$

by setting

$$
h(x, t)=t \cdot f g(x)+(1-t) \cdot x, \quad x \in\left|K^{\prime}\left(U_{L}, L\right)\right|, t \in I .
$$

Clearly if the definition of $h$ is meaningful, then $h$ is a homotopy connecting $f g$ and the identity. In order to show that the definition makes sense, we must show that for any $x \in\left|K^{\prime}\right|$ there is a simplex in $K$ 
whose geometric realization contains both $x$ and $f g(x)$. To see this, let $\sigma=\left\langle V_{1}, \cdots, V_{n}\right\rangle$ be a simplex of $K^{\prime}$ whose geometric realization contains $x$. Suppose the $V^{\prime}$ 's are ordered so that $V_{1} \leqq V_{2} \leqq \cdots \leqq V_{n}$. Let $\tau$ be the simplex of $K$ corresponding to $f g\left(V_{n}\right)$. Since $V_{i} \leqq f g\left(V_{n}\right)$ and $f g\left(V_{i}\right) \leqq f g\left(V_{n}\right)$, by (1), $V_{i} \in|\tau|$ and $f g\left(V_{i}\right) \in|\tau|$ for $i=1,2, \cdots, n$. Hence, $|\sigma| \subseteq|\tau|$ and since $f$, as a map from $\left|K^{\prime}\right|$ to $|K|$, is linear, $f g[|\sigma|] \subseteq|\tau|$. In particular $x \in|\tau|$ and $f g(x) \in|\tau|$. Hence, $f g \sim 1$. Similarly $g f \sim 1$.

Inductive step: $p(C, L)>1$. The proof of the inductive step is based upon the following lemma which will be proved at the end of the paper.

Lemma 2. Let $X \cup Y$ and $Z \cup W$ be simplicial complexes such that $X, Y, Z, W$ are subcomplexes. Let $F: X \cup Y \rightarrow Z \cup W$ be a simplicial map such that

$$
F[X] \subseteq Z, \quad F[Y] \subseteq W
$$

and also such that

$$
\begin{aligned}
& F_{1}=F \mid X: X \rightarrow Z, \\
& F_{2}=F \mid Y: Y \rightarrow W, \\
& F_{3}=F \mid X \cap Y: X \cap Y \rightarrow Z \cap W,
\end{aligned}
$$

are homotopy equivalences. Then $F$ is a homotopy equivalence.

Since $p(C, L)>1$ there is an $x \in V_{L}-C$. Let

$$
\begin{aligned}
& L_{1}=L-\{x\}, \\
& L_{2}=\{y \in L: y \geqq x\} .
\end{aligned}
$$

The partial order on $L$ induces partial orders on $L_{1}$ and $L_{2}$ in which $L_{1}$ and $L_{2}$ are lattices. The zero of $L_{2}$ is $x$. Let $C_{2}=C \cap L_{2}$. Let $\sigma$ be the closed simplex on $C_{2}$, i.e., the simplicial complex whose vertices are the members of $C_{2}$ and whose simplices are all subsets of $C_{2}$. Similarly let $\tau$ be the closed simplex on $U_{L_{2}}$. Then $\sigma \subseteq K(C, L)$ and $\tau \subseteq K\left(U_{L_{2}}, L\right)$. It is easily verified that

$$
\begin{aligned}
K(C, L) & =K\left(C, L_{1}\right) \cup \sigma, \\
K\left(U_{L}, L\right) & =K\left(U_{L}, L_{1}\right) \cup \tau, \\
K\left(C_{2}, L_{2}\right) & =K\left(C, L_{1}\right) \cap \sigma, \\
K\left(U_{L_{2}}, L_{2}\right) & =K\left(U_{L}, L_{1}\right) \cap \tau,
\end{aligned}
$$

and 
(13) $f_{C, L}|| K\left(C, L_{1}\right)|\cap| \sigma \mid=f_{C_{2}, L_{2}}$.

Set $\left|K\left(C, L_{1}\right)\right|=X,|\sigma|=Y,\left|K\left(U_{L}, L_{1}\right)\right|=Z$ and $|\tau|=W$. Let $F=f_{C, L}$. Then $F$ maps $X \cup Y$ to $Z \cup W$, by (6) and (7), and $F, X, Y$, $Z, W$ satisfy (2), by (10) and (11). Since $p\left(C, L_{1}\right)<p(C, L)$ and $p\left(C_{2}, L_{2}\right)<p(C, L)$ it follows from the inductive hypothesis that $f_{C, L_{1}}$ and $f_{C_{2}, L_{2}}$ are homotopy equivalences. Hence by (12), $F_{1}$ (defined by (3)) is a homotopy equivalence. Also by (8), (9), and (13), $F_{3}$ (defined by (5)) is a homotopy equivalence. Finally $Y=|\sigma|$ and $W=|\tau|$ are contractible, so $F_{2}$ is a homotopy equivalence. Hence, by Lemma $3, F=f_{C, L}$ is a homotopy equivalence.

Proof of Lemma 2. Let $G: P \rightarrow Q$ be any continuous map. Let $M(G)$ denote the mapping cylinder of $G$, namely $(P \times I) \cup Q / E$ where $E$ is the equivalence relation generated by the relations $\langle x, 1\rangle E G(x)$ for all $x \in P$. To prove Lemma 2 we will need the following lemma.

Lemma 3. If $G: P \rightarrow Q$ is a cellular map of $\mathrm{CW}$ complexes then $G$ is a homotopy equivalence if and only if $P \times\{0\}$ is a deformation retract of $M(G)$.

To say $P \times\{0\}$ is a deformation retract of $M(G)$ is to say that there is a deformation retraction of $M(G)$ on $P \times\{0\}$, i.e., a map

$$
H: M(G) \times I \rightarrow M(G)
$$

such that

$$
\begin{array}{ll}
H(x, 0)=x, & x \in M(G), \\
H(x, t)=x, & x \in P, \quad t \in I, \\
H(x, 1) \in P, & x \in M(G) .
\end{array}
$$

The "if" part of Lemma 3 is straightforward. The "only if" part follows from the fact that if $G$ is a homotopy equivalence then $\pi_{r}(M(G)$, $P \times\{0\})=0, r=1,2, \cdots$, by the homotopy exact sequence, and from Theorem 1.7 of Chapter VII of [2] and the comment following it.

Returning to the proof of Lemma 2, it follows from Lemma 3 that there is a deformation retraction $H_{3}$ of $M\left(F_{3}\right)$ on $(X \cap Y) \times\{0\}$. By two applications of the homotopy extension property of $\mathrm{CW}$ complexes it follows that there is an extension 


$$
H_{3}^{\prime} ; M(F) \times I \rightarrow M(F)
$$

of $H_{3}$ such that

$$
\begin{aligned}
& H_{3}^{\prime}(x, 0)=x, \quad x \in M(F), \\
& H_{3}^{\prime}(x, t)=x, \quad x \in(X \cup Y) \times\{0\}, \quad t \in I, \\
& H_{3}^{\prime}\left(M\left(F_{1}\right) \times I\right) \subseteq M\left(F_{1}\right), \\
& H_{3}^{\prime}\left(M\left(F_{2}\right) \times I\right) \subseteq M\left(F_{2}\right) .
\end{aligned}
$$

By Lemma 3 there are deformation retractions $H_{1}$ of $M\left(F_{1}\right)$ on $X \times\{0\}$ and $H_{2}$ of $M\left(F_{2}\right)$ on $Y \times\{0\}$. We define a homotopy $H$ : $M(F) \times I \rightarrow M(F)$ by

$$
\begin{aligned}
& H(x, t)=H_{3}^{\prime}(x, 2 t), \quad x \in M(F), \quad 0 \leqq t \leqq 1 / 2, \\
& H(x, t)=H_{1}\left(H_{3}^{\prime}(x, 1), 2 t-1\right), \quad x \in M\left(F_{1}\right), \quad 1 / 2 \leqq t \leqq 1, \\
& H(x, t)=H_{2}\left(H_{3}^{\prime}(x, 1), 2 t-1\right), \quad x \in M\left(F_{2}\right), \quad 1 / 2 \leqq t \leqq 1 .
\end{aligned}
$$

It is easily verified that $H$ is well defined and is a deformation retraction on $(X \cup Y) \times\{0\}$. Hence, by Lemma $3, H$ is a homotopy equivalence.

\section{BIBLIOGRAPHY}

1. C. H. Dowker, Homology groups of relations, Ann. of Math. 56 (1952), 84-95.

2. J. Folkman, The homology groups of a lattice, Rand Corp. Report, 1964.

3. P. J. Hilton, An introduction to homotopy theory, Cambridge Univ. Press, Canıbridge, 1953.

4. G.-C. Rota, On the foundations of combinatorial theory. I, Z. Wahrscheinlichkeitstheorie und Verw. Gebiete 2 (1964), 340-368.

Princeton University 